\title{
Oncogenes, Protein-Kinase
}

National Cancer Institute

\section{Source}

National Cancer Institute. Oncogenes, Protein-Kinase. NCI Thesaurus. Code C18343.

Human Protein-Kinase Oncogenes are mutated variants of Kinase Family Genes, which encode diverse Protein Kinases. Protein kinase enzymes catalyze the conversion of a protein to a phosphoprotein by covalently transferring the terminal, gamma phosphate group from ATP to a variety of substrates that typically play key regulatory roles in diverse cellular functions. Protein-Kinase oncogenes disrupt normal cell function. 